\title{
CCFM Evolution with Unitarity Bound
}

\author{
Emil Avsar \\ Penn State University, University Park, PA 16802, USA \\ We perform a detailed study of the CCFM evolution, complemented with an absorptive \\ boundary which mimics the non-linear dynamics of parton saturation. We present results \\ for the evolution of the unintegrated gluon density in the presence of saturation and extract \\ the energy dependence of the emerging saturation momentum. We show that CCFM and \\ BFKL evolution lead to almost identical predictions after including the effects of gluon \\ saturation and of the running of the coupling.
}

\section{Introduction}

In a series of studies, $[1,2]$, we have proposed a method for effectively implementing saturation and unitarity within a generic linear evolution equation for the unintegrated gluon distribution, so like the BFKL [3] and the CCFM [4] equations. In these proceedings we shall summarise some of the results from [2] and we shall discuss prospects for the future and open questions. For details of the topics to be presented here we refer to the cited articles.

At HERA, for not too low $Q^{2}$ values, schemes based on the NLO DGLAP evolution have been quite successful at describing data. However, the situation at lower $Q^{2}$ is not so clear. A very recent study [5] indeed points to some deviations from NLO DGLAP predictions in HERA data, for smaller $x$ values. One should, however, realise that deviations from the standard collinear approach may be seen even at relatively large momenta $Q$, well above $Q_{s}$. Interesting observables are in particular forward jets, or Mueller-Navelet jets. At LHC, due to the high collisions energy, even events with large $Q^{2}$ can contain effects of small $x$ evolution. Also interesting is the description of angular (de)correlations in multi-jet events.

The search for non-linear effects in data has so far mostly concentrated on inclusive observables, like $F_{2}$ and $F_{L}$, which are generally poor in discriminating between various models (though the use of both of them simultaneously gives stronger constraints). One has moreover mostly used simple dipole models, which contain few fit parameters, but also lack in dynamics since for example energy dependence is fitted by hand. Obviously, it would be a great advantage to be able to study more exclusive observables, as this would make the various predictions from the different dynamics harder to mask via fit parameters or input distributions. Secondly, it is desirable to have an approach which includes non-linear effects and at the same time contains more dynamical information on the underlying evolution. Also, it is by now rather well-known that kinematical effects such as energy conservation are significant. As such effects are hard to include in the analytical formalism, it is an advantage to have a numerical procedure where they can be included together with the small- $x$ evolution and the non-linear effects related to parton saturation.

Our strategy is to enforce an absorptive boundary condition at low transverse momenta which prevents the gluon phase-space occupation numbers to grow beyond their physical values 


\section{CCFM EVOLUTION WITH UNITARITY BOUND}

at saturation. The method is the extension of a strategy originally introduced in relation with analytic studies of the BFKL evolution in the presence of saturation [6, 7]. Importantly, in [1] we demonstrated the power of this method, and its efficiency by comparing the numerical solutions to the BFKL equation with the absorptive boundary condition against those of the non-linear Balitsky-Kovchegov (BK) equation $[8,9]$. We have shown that the absorptive boundary method successfully reproduces the results of the BK equation for both fixed and running coupling, and for all the energies, not only the asymptotic ones. Therefore, the simplicity and efficiency of this method makes it a very compelling tool for phenomenological studies at LHC. In addition, the great advantage is that it can directly be implemented in present Monte Carlo event generators, such as CASCADE [10] and LDCMC [11]. Thus one does not need to write new and complicated codes but can simply modify existing ones.

Of course, being such a simple method, our approach has some drawbacks. One is that one is limited studying the region above $Q_{s}$ where the saturation effects are harder to identify. Below $Q_{s}$ one would need to take into the full non-linear dynamics, which also includes complex manybody correlations and cannot be treated with standard pdfs. Even though saturation certainly plays a greater role in the small momenta region, covering the underlying event at LHC, the analysis of it will be extremely complex and difficult to study. Besides, for such low momenta, it may be difficult to separate saturation physics from the genuinely non-perturbative physics. By staying in the higher $Q$ region we can study saturation and small $x$ effects in the more clean environment of perturbative QCD.

\section{Short Overview of CCFM}

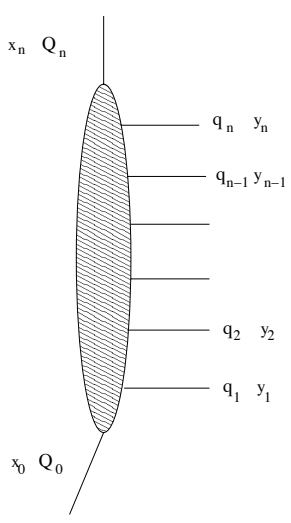

Figure 1: Kinematics of the gluon radiation. The last $t$-channel propagator is denoted by $Q_{n}$, while the real $s$-channel gluons are represented by the horizontal lines.

In Figure 1 we define the kinematics of the evolution. The figure represents a gluon ladder as produced by CCFM where by $q_{i}$ we denote the transverse momenta of the real, $s$-channel, gluons, and we define $Q_{i}=-\sum_{k}^{i} q_{k}$ where we have assumed the incoming virtual, $t$-channel gluon to be collinear with the proton (not shown in the figure). The important aspects of CCFM can be summarised as follows.

Only those diagrams are kept which give the leading factors of $\alpha_{s} \ln s$ where $s$ is the cms 
energy. This implies a strong ordering in the energy of the real gluons:

$$
x=x_{n} \ll y_{n} \ll y_{n-1} \ll \cdots \ll y_{2} \ll y_{1} \approx 1,
$$

where by $y_{i}$ we denote the energy fraction of the $i$ th emitted real gluon. The real gluons are distributed with the standard logarithmic weights $\bar{\alpha}_{s} d q_{i}^{2} / q_{i}^{2} \cdot d y_{i} / y_{i}$. In BFKL the $t$-channel propagators have precisely momenta $Q_{i}$ since there the energy ordering agrees with the order of emission. In CCFM, however, the real gluon emissions are instead ordered according to their angles $\xi_{i} \equiv q_{i}^{2} /\left(y_{i}^{2} E^{2}\right)$ ( $E$ is the energy of the proton). However, it turns out that also in CCFM can one approximately identify the $Q_{i}$ as the momenta of the $t$-channel propagators. This is crucial in rewriting CCFM in the form which is commonly used.

In addition there are in CCFM two types of virtual corrections which both exponentiate, $S_{e i k}^{2}$ and $S_{n e}^{2}$. They are related to corrections involving the eikonal and non-eikonal components of the three-gluon vertex. As shown in [2] one has at each vertex $k$

$$
S_{n e}^{2}(k) \cdot S_{e i k}^{2}(k)=\Delta_{n e}^{B F K L}(k) .
$$

where $\Delta_{n e}^{B F K L}(k)$ is the so-called "non-Sudakov" form factor in BFKL.

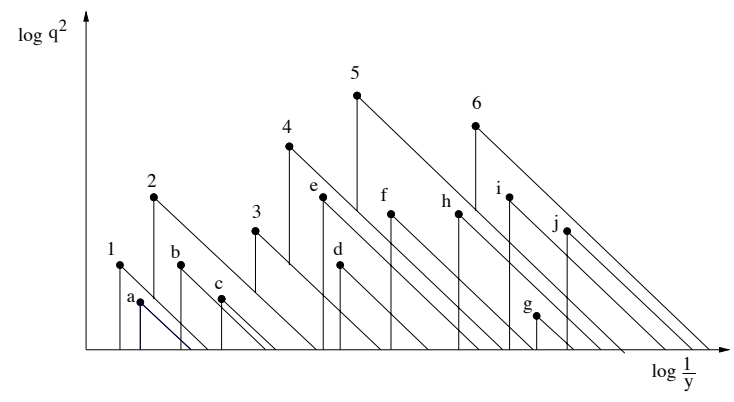

Figure 2: Dividing the CCFM radiation into two subsets: hard emissions (enumerated emissions) and soft emissions (indexed by small letters) All real emissions are represented by fat dots. The vertical axis denote the transverse momenta while the horizontal axis the inverse energy fraction. The diagonal lines indicate the angles of the emissions. One sees that soft emissions also have small transverse momenta.

A very important feature of the CCFM cascade is the identification of so called soft and hard emissions in the ladder. The hard emissions are those real emissions which are ordered in both energy and angle, while the soft emissions are such that they are always followed in the angular ordering by a gluon with higher energy. If the ladder consisted only of hard emissions, the $t$-channel propagators in CCFM would have momenta exactly equal to $Q_{i}$ as then the angular and the energy orderings coincide. However, the soft emissions which break the exact correspondence are soft not only in energy but also in momenta, see Figure 2. This implies that they, to a first approximation, do not change the momenta of the virtual propagators. Therefore one can indeed identify the $Q_{i}$ with the propagator momenta, even in the angular ordered cascade. This argument shows that there is an implicit approximation in the CCFM formalism - the fact that soft gluon emissions are assumed not to change the virtual transverse momenta. The reason for classifying the emissions as hard and soft is because it allows one to rewrite the gluon distribution in a much simplified form. 


\section{CCFM EVOLUTION WITH UNITARITY BOUND}

Within these approximations, the CCFM evolution equation for the gluon density can be rewritten in three different levels. The first one is the most exclusive one including all ${ }^{1}$ soft and hard emissions from the gluon ladder. It is given by

$$
\begin{array}{r}
\mathcal{A}(x, k, \bar{p})=\bar{\alpha}_{s} \int_{x}^{1} d z \int \frac{\mathrm{d}^{2} p}{\pi p^{2}} \theta(\bar{p}-z p) \Delta_{s}(\bar{p}, z p)\left(\frac{\Delta_{n s}(k, z, p)}{z}+\frac{1}{1-z}\right) \\
\times \mathcal{A}\left(\frac{x}{z},|k+(1-z) p|, p\right) .
\end{array}
$$

Here we are using rescaled momenta within the integrand: $\bar{p}=\bar{q} /(1-x)$ and $p=q /(1-z)$. The third argument of the gluon distribution indicates the maximally allowed emission angle, $\bar{\xi}=\bar{q}^{2} /\left(x^{2} E^{2}\right)$. This is a feature specific to CCFM and follows from the coherence property of the emissions. The $1 / z$ piece comes from the hard emissions while $1 /(1-z)$ comes from the soft emissions. Now, the soft emissions can be exactly compensated by the Sudakov form factors $\Delta_{s}$ so that this expression is equivalent to the more inclusive expression

$$
\mathcal{A}(x, k, \bar{p})=\bar{\alpha}_{s} \int_{x}^{1} \frac{\mathrm{d} z}{z} \int \frac{\mathrm{d}^{2} p}{\pi p^{2}} \theta(\bar{p}-z p) \Delta_{n s}(k, z, p) \mathcal{A}\left(\frac{x}{z},|k+(1-z) p|, p\right)
$$

which includes only the hard emissions explicitly. It does not make sense to say one of the equations is preferred to the other, within the approximations of CCFM they are equivalent. Thus one could base an event generator on (4) to construct the gluon distribution but then the soft emissions must be later included a final state emissions to get the correct final state. Nevertheless, in practice we expect the two equations also to give different gluon distributions, see the discussion below.

In deriving the integral equations (and also $\Delta_{n s}$ and $\Delta_{s}$ out of $S_{n e}^{2}$ and $S_{e i k}^{2}$ ), the approximation that the soft emissions do not change the propagator momenta was used. However, there are also a class of hard emissions which are, in transverse momenta, as "soft" as the soft emissions. Within the approximations of CCFM, these hard emissions also do not change the propagator transverse momenta. As was realised first in [12], those hard emissions can be used to cancel the non-Sudakov, $\Delta_{n s}$, simplifying the evolution equation even more. There are, however, two caveats. The first is that this is only possible if the kinematical constraint $k^{2}>z q^{2}$ is explicitly introduced ${ }^{2}$. Secondly the explicit definition of the transverse momenta conserving hard emissions requires a cut directly in momentum space, using a theta function in the momentum variables. This is contrast with the constraint put on the soft emissions, which is not directly written in momentum space. This results in a certain ambiguity since this cut can within the approximations be chosen somewhat differently. This implies that there is no unique equation to derive out of (4). The explicit choice for example used in the LDC model [12] (and this was also one of the choices in [2]) leads to the equation

$$
\begin{aligned}
& \partial_{Y} \mathcal{A}(Y, k)=\bar{\alpha}_{s} \int \frac{\mathrm{d} k^{\prime 2}}{\left|k^{2}-k^{\prime 2}\right|} h(\kappa)\left(\theta\left(k^{2}-k^{\prime 2}\right) \mathcal{A}\left(Y, k^{\prime}\right)\right. \\
& \left.+\theta\left(k^{\prime 2}-k^{2}\right) \theta\left(Y-\ln \left(k^{\prime 2} / k^{2}\right)\right) \mathcal{A}\left(Y-\ln \left(k^{\prime 2} / k^{2}\right), k^{\prime}\right)\right) .
\end{aligned}
$$

\footnotetext{
${ }^{1}$ These are not all the emissions in the final state however. There are further emissions from the $s$ channel gluons and these have be taken into account for a faithful description of the exclusive final state.

${ }^{2}$ As a matter of fact, the original article [13] mentions that this constraint should indeed be included.
} 
where

$$
h(\kappa)=1-\frac{2}{\pi} \arctan \left(\frac{1+\sqrt{\kappa}}{1-\sqrt{\kappa}} \sqrt{\frac{2 \sqrt{\kappa}-1}{2 \sqrt{\kappa}+1}}\right) \theta(\kappa-1 / 4) .
$$

and $\kappa \equiv \min \left(k^{2}, k^{\prime 2}\right) / \max \left(k^{2}, k^{2}\right)$. A different choice of the explicit cut leads instead to

$$
\begin{aligned}
& \partial_{Y} \mathcal{A}(Y, k)=\bar{\alpha}_{s} \int \frac{\mathrm{d} k^{\prime 2}}{\max \left(k^{2}, k^{\prime 2}\right)}\left(\theta\left(k^{2}-k^{\prime 2}\right) \mathcal{A}\left(Y, k^{\prime}\right)\right. \\
& \left.+\theta\left(k^{\prime 2}-k^{2}\right) \theta\left(Y-\ln \left(k^{\prime 2} / k^{2}\right)\right) \mathcal{A}\left(Y-\ln \left(k^{\prime 2} / k^{2}\right), k^{\prime}\right)\right) .
\end{aligned}
$$

One should immediately notice that $\mathcal{A}$ now only depends on $x$ and $k$, the dependence on the third variable $\bar{q}$ has dropped out. As explained in [2] this is really only true when $k \lesssim \bar{q}$. Thus strictly speaking these last equations are not valid when $k$ is larger than $\bar{q}$.

\section{CCFM with Absorptive Boundary}

Here we shall not discuss the theoretical motivations for using the absorptive boundary method in studying the non-linear effects upon the evolution (for this we refer again to [2]) but we shall rather simply explain how it is implemented.

We first define the logarithmic variables $\rho=\ln k^{2} / k_{0}^{2}$ and $Y=\ln 1 / x$. Introduce a line of constant gluon occupancy $\rho=\rho_{c}(Y)$ via the condition

$$
\mathcal{A}\left(Y, \rho=\rho_{c}(Y)\right)=c,
$$

where the number $c$ is smaller than one, but not much smaller. The saturation line $\rho_{s}(Y)$ would correspond to $c \sim 1$, so clearly $\rho_{s}(Y)$ is smaller than $\rho_{c}(Y)$, but relatively close to it. For $\rho<\rho_{c}(Y)$ and sufficiently high energy, the solution $\mathcal{A}(Y, \rho)$ would become arbitrarily large. If this equation is to be solved numerically, one may think about enforcing saturation in the following way: at each step in $Y$, one first identifies the corresponding point $\rho_{c}(Y)$ from the condition (8), and then one requires $\mathcal{A}(Y, \rho)$ to remain finite and of $\mathcal{O}(1)$ for any $\rho$ sufficiently far below $\rho_{c}(Y)$ - say, for $\rho \leq \rho_{c}(Y)-\Delta$ with $\Delta \simeq \ln (1 / c)$. When decreasing $\rho$ below $\rho_{c}(Y)$, the solution $\mathcal{A}(Y, \rho)$ will typically start by rising, then reach a maximum and eventually decrease to the finite value determined by hand. The saturation scale $\rho_{s}(Y)$ can then be conventionally identified with the position of this maximum. In this procedure, the numbers $c$ and $\Delta$ are to be viewed as free parameters, which are however correlated with each other as $\Delta \sim \ln (1 / c)$. In this way the energy dependence of the saturation scale follows from the dynamics, and is thus not fitted by hand, and is guaranteed to be consistent with evolution.

In Figure 3 we plot the respective predictions of (5) and BFKL for both fixed and running coupling. To make the comparison between the solutions easier, we have scaled the solutions to BFKL so that the solutions more or less coincide at $Y=10$. For fixed coupling, the saturation front generated by (5) is seen to progress significantly faster than the one generated by BFKL. For the running coupling case on the other hand, it is clear that the respective fronts progress with similar speeds. Thus in this case the two equations have similar predictions for the energy dependence of the saturation momentum, so that the entire difference between them 


\section{CCFM EVOLUTION WITH UNITARITY BOUND}
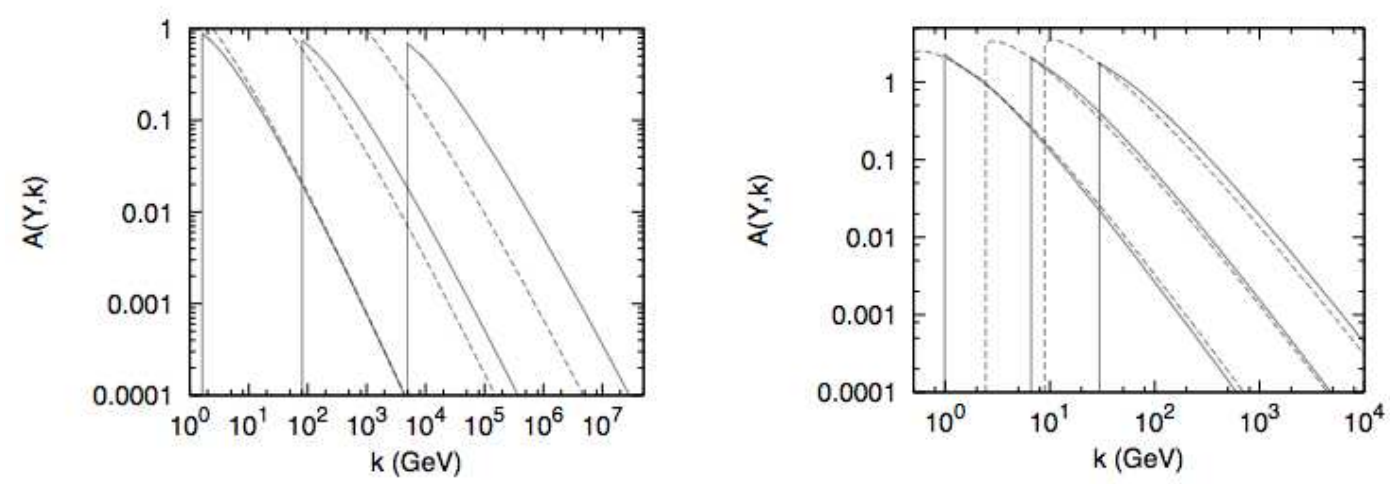

Figure 3: The solutions to equations (5) (solid lines) and BFKL (dashed lines) including the absorptive boundary, for $Y=10,20,30$. The solutions to BFKL includes the kinematical constraint and have been scaled so that the solutions match at $Y=10$. Left: fixed coupling $\alpha_{s}=0.2$. Right: running coupling.

can (almost) be removed by either a rescaling of the saturation momentum $Q_{s}$, or as in the figure, a rescaling of $\mathcal{A}$.

In Figure 4 (left) we show the respective results for the running coupling case for much higher values of $Y$. Also, in the right figure there, we compare the absorptive boundary results for (5) and (7). One clearly sees now that all the equations under consideration give approximately the same speed for the saturation front. Note that in the left figure the solutions to BFKL have been scaled to make the comparison easier. From the right figure we see that (5) and (7) roughly have the same shape and energy dependence when saturation and a running coupling is introduced. This is big contrast to the linear case (fixed or running coupling) and also to the non-linear case with a fixed coupling, where the differences between the solutions is much larger and grows with energy. Thus it seems that the inclusion of saturation and a running coupling removes much of the ambiguity inherent in the derivation of the different versions of CCFM.

From our results in [1] and [2] we also see that the solutions to the gluon distribution with saturation differs from the linear solutions quite substantially even for moderate values of $Y$ reachable at the LHC. This, however, does not necessarily imply that the effects of the absorptive boundary will be clearly visible in phenomenological applications. First one should remember that there are many more effects in such applications which slow down the growth of the gluon distribution with $Y$, most notably energy-momentum conservation which gives large corrections. Secondly, even in the application of linear equations, such as CCFM in the CASCADE event generator, there is included an absorptive boundary in $k$. This is however not a boundary in the sense of the one presented here, which has its motivation in the perturbative dynamics of non-linear evolution, but it is rather a soft cut which is fixed for all energies. It is therefore more of a non-perturbative cut. However in the limited $Y$ interval covering phenomenologically relevant energies, it may be difficult to distinguish the effects of the different boundaries.

In addition to these issues, we must say that there are further issues with CCFM not very well understood. One is the fact that non-leading modifications seem to have huge effects. This makes the whole evolution questionable as one can switch different components, within the approximations of the formalism, and get very different results. One can for example 

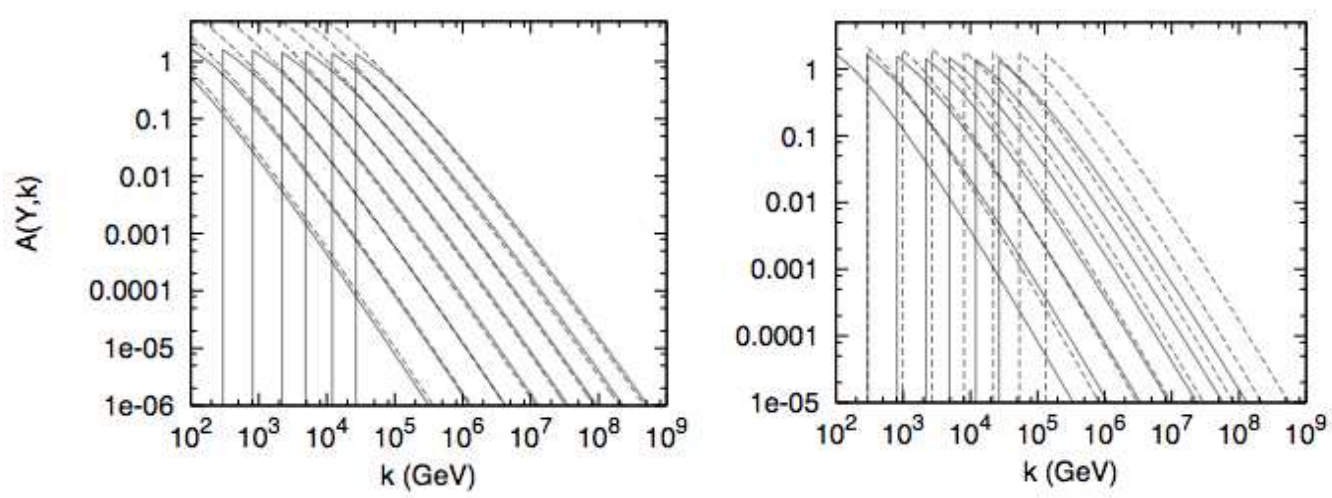

Figure 4: Solutions to (5), (7) and BFKL including the saturation boundary for a running coupling. Left: (5) (dashed lines) and BFKL (solid lines). The solution to BFKL has been scaled by a factor 6.5. Y between 50 and 140 units. Right: (5) (solid lines) and (7) (dashed lines). $Y$ between 40 and 100 units.

get results where the growth of the gluon density sets in very late, at $x$ values below $10^{-5}$ [14]. A notable difficulty of CCFM is also that it appears to fail in the description of forward jets, precisely due to the non-leading terms which are otherwise needed to fit the structure function. We believe that it may be premature to try seriously study CCFM with saturation for phenomenological studies before these issues are resolved. It may on the other hand be that saturation removes many of the ambiguities of the formalism, some of which we have already seen. Together with this we believe that the next step in our analysis is to study equations (3) or (4) with the absorptive boundary and compare those results to see whether the evolution is different or not. Notice that an interesting and immediate consequence of such a study will be that the saturation momentum $Q_{s}$ will now depend not only on $x$ but also on the momentum scale $\bar{q}$ (which in DIS is roughly $Q^{2}$ ). This is again due to the coherence and implies that the scale when the gluon fields in the proton saturates depends not only intrinsically on the proton but also on the probe, certainly an interesting result.

\section{Acknowledgements}

The work presented here was performed while I was a postdoc at CEA/Saclay, I would like to thank E. Iancu for this collaboration and also for all the help during my time in Saclay. I would also like to thank G. Gustafson for useful discussions. I am also grateful to H. Jung for many e-mail exchanges and also many discussions.

\section{References}

[1] Emil Avsar and Edmond Iancu. BFKL and CCFM evolutions with saturation boundary. Phys. Lett., B673:24-29, 2009.

[2] Emil Avsar and Edmond Iancu. CCFM Evolution with Unitarity Corrections. Nucl. Phys., A829:31-75, 2009. 


\section{CCFM EVOLUTION WITH UNITARITY BOUND}

[3] L.N. Lipatov, Sov. J. Nucl. Phys. 23 (1976) 338;

E.A. Kuraev, L.N. Lipatov and V.S. Fadin, Zh. Eksp. Teor. Fiz 72, 3 (1977) (Sov. Phys. JETP 45 (1977) 199);

Ya.Ya. Balitsky and L.N. Lipatov, Sov. J. Nucl. Phys. 28 (1978) 822.

[4] Catani, S. and Fiorani, F. and Marchesini, G. , Nucl. Phys. B336 (1990) 18; Ciafaloni, M. Nucl. Phys. B296 (1988) 49;

Catani, S. and Fiorani, F. and Marchesini, G. , Phys. Lett. B234 (1990) 339.

[5] Fabrizio Caola, Stefano Forte, and Juan Rojo. Deviations from NLO QCD evolution in inclusive HERA data. 2009.

[6] Edmond Iancu, Kazunori Itakura, and Larry McLerran. Geometric scaling above the saturation scale. Nucl. Phys., A708:327-352, 2002.

[7] A. H. Mueller and D. N. Triantafyllopoulos. The energy dependence of the saturation momentum. Nucl. Phys., B640:331-350, 2002.

[8] I. Balitsky. Operator expansion for high-energy scattering. Nucl. Phys., B463:99-160, 1996.

[9] Yuri V. Kovchegov. Small-x F2 structure function of a nucleus including multiple pomeron exchanges. Phys. Rev., D60:034008, 1999.

[10] H. Jung and G. P. Salam. Hadronic final state predictions from CCFM: The hadron- level Monte Carlo generator CASCADE. Eur. Phys. J., C19:351-360, 2001.

[11] Hamid Kharraziha and Leif Lönnblad. The linked dipole chain Monte Carlo. JHEP, 03:006, 1998.

[12] Bo Andersson, G. Gustafson, and J. Samuelsson. The linked dipole chain model for dis. Nucl. Phys., B467:443-478, 1996.

[13] S. Catani, F. Fiorani, and G. Marchesini. Small x Behavior of Initial State Radiation in Perturbative QCD. Nucl. Phys., B336:18, 1990.

[14] G. Bottazzi, G. Marchesini, G. P. Salam, and M. Scorletti. Small-x one-particle-inclusive quantities in the CCFM approach. JHEP, 12:011, 1998. 